\title{
Multidetector computed tomography analysis of benign and malignant nodules in patients with chronic lymphocytic thyroiditis
}

\author{
CAISONG ZHU ${ }^{1 *}$, WEI LIU ${ }^{1 *}$, JUN YANG $^{1}$, JING YANG $^{2}$, KANGWEI SHAO $^{1}$, \\ LIXIN YUAN ${ }^{1}$, HAIRONG CHEN ${ }^{1}$, WEI LU ${ }^{1}$ and YING ZHU ${ }^{1}$ \\ Departments of ${ }^{1}$ Radiology and ${ }^{2}$ Pathology, Tongren Hospital, \\ Shanghai Jiao Tong University School of Medicine, Shanghai 200336, P.R. China
}

Received March 20, 2015; Accepted April 8, 2016

DOI: $10.3892 / \mathrm{ol} .2016 .4585$

\begin{abstract}
The aim of the present study was to compare the multidetector computed tomography (MDCT) features of benign and malignant nodules in patients with chronic lymphocytic thyroiditis (CLT). MDCT findings, including the size, solid percentage, calcification, margin, capsule, anteroposterior-transverse diameter ratio as well as the mode and the degree of enhancement of 137 thyroid nodules in 127 CLT cases were retrospectively analyzed. Furthermore, the correlation between MDCT findings and pathological results combined with the CT perfusion imaging was analyzed for the differences between benign and malignant nodules. A total of $77.5 \%$ (31/40) of malignant nodules were completely solid, and 33\% (32/97) of benign nodules were predominantly cystic. Compared with the benign nodules, micro-calcification and internal calcification were more frequently observed in the malignant nodules $(\mathrm{P}<0.05)$. MDCT features such as ill-defined margin, absence of capsule or incomplete capsule or homogeneous enhancement were more likely to be present in the malignant nodules $(\mathrm{P}<0.05)$. Nevertheless, no significant difference was observed in the enhancement degree at arterial or venous phase between benign and malignant nodules $(\mathrm{P}>0.05)$. MDCT features are useful in differentiating the benign and malignant nodules in CLT patients, and it may be essential for a radiologist to review the MDCT characteristics of nodules in the clinical practice.
\end{abstract}

\section{Introduction}

Chronic lymphocytic thyroiditis (CLT), also termed Hashimoto thyroiditis, is a type of autoimmune disease characterized by

Correspondence to: Dr Jun Yang, Department of Radiology, Tongren Hospital, Shanghai Jiao Tong University School of Medicine, 1111 Xianxia Road, Shanghai 200336, P.R. China

E-mail: junyangy@163.com

*Contributed equally

Key words: multi-slice computed tomography, thyroid nodules, chronic lymphocytic thyroiditis thyroid antigen response and $\mathrm{T}$ and $\mathrm{B}$ lymphocytes infiltration $(1,2)$. Generally, 95\% of CLT cases occur in women, particularly among women between 30 and 50 years of age (3). Thyroid nodule is a common disease found in the thyroid, and CTL usually coexists with benign and malignant thyroid nodules (4-6). In the majority of cases, CTL combined with malignant thyroid nodules was the reason for surgery and most patients underwent surgery following an inconclusive diagnosis (7). Therefore, it may be of significant clinical benefit to identify a method to distinguish between benign and malign thyroid nodules.

Previously, cases of CLT with thyroid nodules were typically diagnosed by ultrasonography $(6,8,9)$ and fine needle aspiration biopsy (FNAB) (10-12). However, there remains a lack of specific sonographic features that provide a prediction value with high sensitivity and high positivity in thyroid, which makes it difficult to differentiate benign nodules from malignant nodules in CLT patients. Yang et al (13) conducted a clinical trial enrolling 1,100 patients and the authors observed that only a specificity of $75 \%$ was confirmed by using thyroid biopsy (13). Notably, a study has recommended the application of multidetector computed tomography (MDCT) in thyroid nodules diagnosis (14). However, MDCT has not previously been used to differentiate the benign from malignant nodules thyroid nodules in CLT patients.

In the present study, 137 thyroid nodules in 127 CLT patients combined with benign and malignant thyroid nodules underwent MDCT perfusion imaging. The present study retrospectively analyzed the characteristics of nodules, including the size, solid percentage, calcification, margin, capsule, anteroposterior-transverse diameter ratio as well as the mode and the degree of enhancement. In addition, the correlation between pathological results and the computed tomography (CT) perfusion imaging was also analyzed for assessing the differences between benign and malignant nodules.

\section{Subjects and methods}

Subjects. A total of 127 CTL patients (10 males and 117 females; median age, 52 years, range 19-77 years) diagnosed with thyroid nodules by palpation or B-mode ultrasound from January 2005 to December 2013 at the Tongren Hospital Affiliated to Shanghai Jiaotong University School of Medicine 
(Shanghai, China) were retrospectively reviewed in the study. The nodules were confirmed as benign or malignant nodules by surgery. In addition, all the patients received an MDCT scan on the thyroid less than a week prior to surgery, and the patients did not receive any treatment during the week.

The present study was approved by the hospital medical ethics committees and written informed consent was obtained from all participants.

MDCT technical parameters. The patients were placed in a supine position with hyperextended neck. All CT perfusion images were obtained using a MDCT scanner (Lightspeed Pro32 CT; GeneralElectric, Milwaukee, WI, USA) with the following parameters: $2.5-\mathrm{mm}$ section thickness and section slice, and 30-35 sec scan delay at an arterial phase while 50-60 sec scan delay at a parenchymal phase. The scanning range was initially performed in a craniocaudal direction from the superior border of hyoid to the aorta arch, including the entire thyroid mass (mean coverage, $250 \mathrm{~mm}$ ). Each patient received $100 \mathrm{ml}$ of nonionic contrast material (iohexol, $300 \mathrm{mg} / \mathrm{ml}$, GE Healthcare) via a peripheral arm vein at a flow rate of $2.5-3 \mathrm{ml} / \mathrm{s}$ and a dose of $1.5 \mathrm{ml} / \mathrm{kg}$. After $2 \mathrm{sec}$, axial images were reconstructed at $1.25-\mathrm{mm}$ increments at the end of the scan and then the coronal section was reconstructed.

Image analysis. Hard copies were analyzed by two radiologists who recorded every nodule with regard to diagnostic confidence and nodule size to correct classification into one of the size classes. Disagreement would be resolved through discussing with another experienced radiologist. The CT features were defined as follows through combining the studies by Kim et al (12) and Kang et al (15): i) Solid vs. cystic: Completely solid (>95\% solid), predominantly solid (50-95\% solid), predominantly cystic (1-49\% solid); ii) calcification: Micro-calcification (diameter $\leq 2 \mathrm{~mm}$ ), macro-calcification (diameter $>2 \mathrm{~mm}$ ), eggshell (arc-shaped or semi arc-shaped) or mixed (coexist with multiple calcification) in morphology; internal or peripheral calcification in position; iii) margins: Well-defined or ill-defined at enhancement stage; iv) capsule: Intact, incomplete, or unclear and none; v) enhancement mode: Completely cystic, not enhanced, homogeneous enhancement or heterogeneous enhancement; vi) lesion size: Maximum diameter of the lesion; vii) the ratio of anteroposterior to transverse diameter; viii) enhancement degree: The net enhancement value at arterial or venous phase was calculated by enhanced CT scan value subtracting plain CT scan value. The measurement of the $\mathrm{CT}$ values should avoid regions presented with cystic change, necrosis, calcification and vessels.

Hematoxylin and eosin (HE) staining. The nodule tissues of the CLT patients were fixed in $10 \%$ formalin and embedded in paraffin. Next, the tissues were cut into 5- $\mu \mathrm{m}$ consecutive sections and stained with HE. The images were observed under a Leica DM2500 fluorescence microscope (Leica Microsystems GmbH, Wetzlar, Germany).

Statistical analysis. All the statistical analyses were performed using SPSS 17.0 software (SPSS, Chicago, IL,
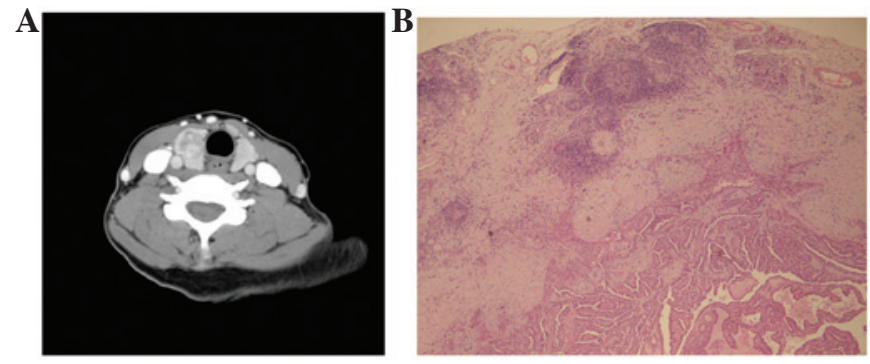

Figure 1. Characteristics of chronic lymphocytic thyroiditis and right lobe papillary cancer by computed tomography pathological microscopy. (A) Enhanced computed tomography suggested cystic degeneration accompanied with enhanced wall nodules with incomplete capsule and incomplete enhanced rings. (B) Papillary growth and extracapsular extension of the cancer cells, the mature lymphocytes infiltration and lymphoid follicular formation in the periphery tissues (hemotoxylin and eosin; magnification, $\mathrm{x} 40$ ).
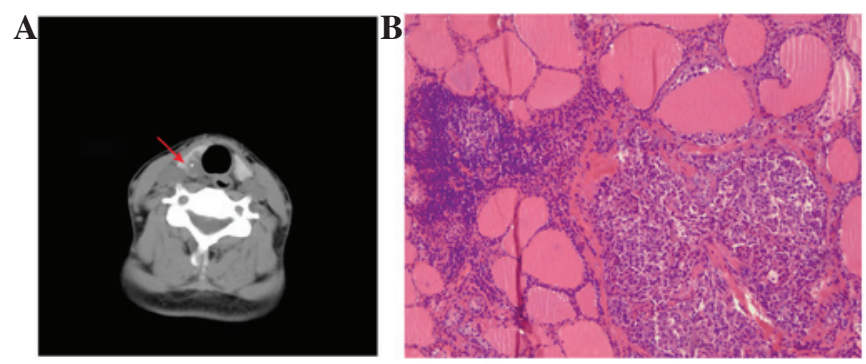

Figure 2. Characteristics of chronic lymphocytic thyroiditis and right lobe medullary cancer by computed tomography pathological microscopy. (A) Plain computed tomography showed low density nodule, ill-defined margin and central micro-calcification (arrow) was found at the right lobe thyroid. (B) Peninsula growth pattern of cancer cells and the cells were isolated by fibrous tissues and amyloid substance, and irregular narrow without capsule was found between cancer cells and adjacent tissues. The mature lymphocytes infiltration and lymphoid follicular formation in the periphery region (hemotoxylin and eosin; magnification, $\mathrm{x} 40$ ).

USA). The Chi-square test was used to analyze the differences among enumeration data. Student's $t$-test was used for data measurement after homogeneity test of variance. A value of $\mathrm{P}<0.05$ was considered to indicate a statistically significant difference.

\section{Results}

Pathological features of the lesions. Among the CTL patients, 40 cases coexisted with malignant nodules, including 26 cases of papillary cancer (Fig. 1A and B), 10 cases of microcarcinoma, 1 case of papillary cancer combined with microcarcinoma, 2 cases of medullary cancer (Fig. 2A and B), and 1 case of lymphoma. Totally, 87 CLT patients coexisted with benign nodules, including 63 cases of follicular adenoma (Fig. 3A and B), 7 cases of nodules goiter and 17 cases of nodules hyperplasia (Fig. 4A and B). In addition, among the 40 patients with malignant nodules, including 3 papillary cancer patients, 1 medullary cancer patient and 5 microcarcinoma patients combined with benign follicular adenoma at contralateral side, were also divided into benign group. Finally, 137 nodes from 127 cases were enrolled in this study, including 97 nodules from benign group and 40 nodules from malignant group. 
Table I. Comparison of multi-slice computer tomography features of benign and malignant nodules.

\begin{tabular}{lccrr}
\hline Nodule characteristic & Benign $(\%)$ & Malignant $(\%)$ & $\chi^{2}$ value & P-value \\
\hline Solid vs. cystic & & & & \\
Completely solid & $32.0(31 / 97)$ & $77.5(31 / 40)$ & 23.709 & $<0.001$ \\
Predominantly solid & $35.0(34 / 97)$ & $22.5(9 / 40)$ & 2.072 & 0.150 \\
Predominantly cystic & $33.0(32 / 97)$ & $0(0 / 40)$ & 17.217 & $<0.001$ \\
Calcifications & & & & 0.013 \\
Incidence & $21.6(21 / 97)$ & $37.5(17 / 40)$ & 6.143 & 0.001 \\
Micro & $5.2(5 / 97)$ & $22.5(11 / 40)$ & 11.629 & 1.000 \\
Macro & $8.2(8 / 97)$ & $7.5(3 / 40)$ & 0.000 & 1.000 \\
Eggshell & $2.1(2 / 97)$ & $2.5(1 / 40)$ & 0.000 & $<0.001$ \\
Mixed & $6.2(6 / 97)$ & $5(2 / 40)$ & 0.000 & $<0.001$ \\
Internal & $19.0(4 / 21)$ & $88.2(15 / 17)$ & 17.989 & $<0.001$ \\
Peripheral & $81.0(17 / 21)$ & $11.8(2 / 17)$ & & $<0.001$ \\
Margins & & & 31.408 & $<1.989$ \\
Well-defined & $76.3(74 / 97)$ & $25.0(10 / 40)$ & 31.408 & 0.002 \\
Ill-defined & $23.7(23 / 97)$ & $75.0(30 / 40)$ & & $<0.001$ \\
Capsule & & & 36.152 & 0.588 \\
Intact & $63.9(62 / 97)$ & $6.5(3 / 40)$ & 25.399 & 0.001 \\
Unclear or none & $36.1(35 / 97)$ & $27.5(11 / 40)$ & & 0.001 \\
Incomplete & $0(0 / 97)$ & $0(0 / 40)$ & 10.228 & 0.108 \\
Enhancement & $21.6(21 / 97)$ & $37.5(15 / 40)$ & 2.579 & \\
Completely cystic & $25.8(25 / 97)$ & & & \\
Homogenous & $52.8(51 / 97)$ & & & \\
Heterogeneous & & & & \\
\hline
\end{tabular}
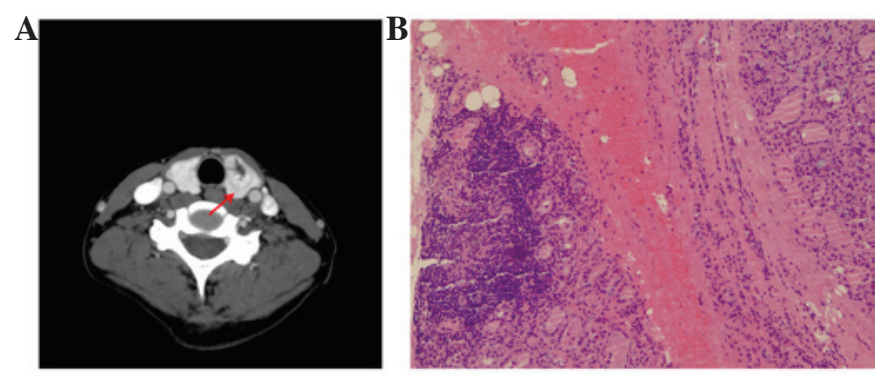

Figure 3. Characteristics of chronic lymphocytic thyroiditis and left lobe follicular adenoma by computed tomography pathological microscopy. (A) Enhanced computed tomography demonstrated peninsula enhancement and central cystic degeneration were observed in one nodule of left lobe. A low density halo (arrows) with well-defined margin is observed in the periphery region. (B) Pathological microscopy demonstrated that intact capsule accompanied with normal follicular pattern, characterized by homogeneous size distribution. The mature lymphocytes infiltration and lymphoid follicular formation in the peripheral region (hemotoxylin and eosin; magnification, $\mathrm{x} 40$ ).

Comparison of benign and malignant nodules within CLT. As demonstrated in Table I, there were significant differences in the CT features, including completely solid, predominantly cystic, calcification rate, micro-calcification, peripheral calcification, internal calcification, well or ill-defined margins, intact or incomplete capsule and homogenous enhancement between benign and malignant group $(\mathrm{P}<0.05)$. Most of the malignant nodules showed completely solid composition (31/40), and
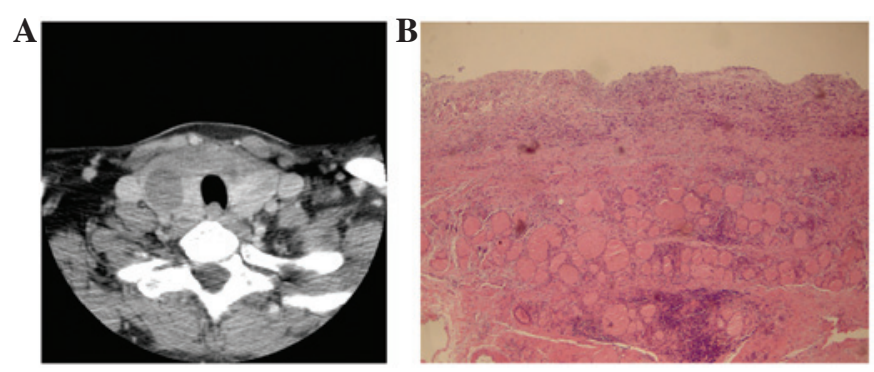

Figure 4. Characteristics of chronic lymphocytic thyroiditis with cystic degeneration by computed tomography pathological microscopy. (A) Enhanced computed tomography demonstrated a diffuse decrease of density in right lobe of the thyroid with a cystic nodule. (B) Pathological microscopy showed that cystic wall formed by fibrous tissues. Mature lymphocytes infiltration was found inside cystic wall in peripheral tissues. No lymphoid follicular formation was observed (hemotoxylin and eosin; magnification, $\mathrm{x} 40$ ).

none of samples among malignant was predominantly cystic. However, only $33 \%$ (32/97) of the predominantly cystic composition were found in benign nodules. Compared with the benign nodules, calcification was more frequently observed in malignant nodules (37.5 vs. $21.6 \%$ ), mainly exhibiting as micro-calcification ( 22.5 vs. $5.2 \%)$ and internal calcification (88.2 vs. $19.0 \%)$. Well-defined $(76.3 \%)$ and intact capsule $(63.9 \%)$ were present in most of benign nodules. There was a trend towards ill-defined margin $(75.0 \%)$, unclear $(65.0 \%)$ or incomplete $(27.5 \%)$ capsule in most of malignant nodules. 
Table II. Comparison of morphological characteristics of benign and malignant nodules (mean \pm standard deviation).

\begin{tabular}{lccccc}
\hline $\begin{array}{l}\text { Classification } \\
\text { of nodules }\end{array}$ & Nodule size $^{\mathrm{a}}$ & $\begin{array}{c}\text { Anteroposterior } \\
\text { and transverse } \\
\text { diameter ratio }\end{array}$ & Plain scan $^{\mathrm{b}}$ & $\begin{array}{c}\text { Net enhancement } \\
\text { degree at arterial } \\
\text { phase }^{\mathrm{b}}\end{array}$ & $\begin{array}{c}\text { Net enhancement } \\
\text { degree at } \\
\text { venous phase }^{\mathrm{b}}\end{array}$ \\
\hline $\begin{array}{l}\text { Benign } \\
\text { Malignancy }\end{array}$ & $15.5 \pm 8.1$ & $1.10 \pm 0.25$ & $71.1 \pm 22.1$ & $86.8 \pm 40.5$ & $48.6 \pm 27.6$ \\
$t$ value & $11.8 \pm 6.1$ & $1.03 \pm 0.14$ & $66.5 \pm 23.1$ & $88.3 \pm 51.4$ & $54.1 \pm 27.5$ \\
P-value & -2.544 & 1.618 & -1.024 & 0.165 & 1.01 \\
\hline
\end{tabular}

${ }^{\mathrm{a}} \mathrm{mm} ;{ }^{\mathrm{b}} \mathrm{HU}$.

Among malignant nodules, 7 presented with incomplete enhanced ring. Among the heterogeneous benign nodules, 16 were peninsula-like enhancement which has irregular low density area in the periphery region or significantly enhanced nodules in the central region, while only 7 patients in the malignant group presented peninsula-like enhancement. In addition, the incidence of malignant nodules was significantly higher than the benign nodules $(55.0$ vs. $25.8 \%, \mathrm{P}<0.05)$ among the homogeneous nodules.

As presented in Table II, the size of the benign nodules was significantly larger than the malignant nodules, but the ratio of anteroposterior to transverse diameter, anteroposterior to net enhancement degree at arterial or venous phase were not significantly different between the two groups $(\mathrm{P}>0.05)$.

\section{Discussion}

Although the association between CLT and thyroid cancer remains controversial (16), emerging evidence has demonstrated that there is an increased risk of papillary thyroid carcinoma for patients with CLT (17-19). In the present study, among patients with CLT, the incidence of malignancy was $31.5 \%$ (40/127), including 26 papillary cancer cases and 11 microcarcinoma cases. Notably, malignant nodules were normally characterized by having a completely solid composition, internal calcification, ill-defined margin, an unclear or incomplete capsule, while benign nodules were more likely to have peripheral calcification, well-defined margins and an intact capsule. In addition, the size of the benign nodules was significantly larger than the malignant nodules.

In accordance with the findings by MDCT, sonographic analysis of benign and malignant nodules in diffuse hashimoto thyroiditis patients has demonstrated that malignant nodules were more likely to be solid and hypoechoic (1). In addition, Hashimoto's thyroiditis cases associated with thyroid nodular disease were also represented by solid composition, hypoechogenicity and micro-calcifications (6). A previous study by Kim et al (12) reported the sonographic characteristics of micro-calcifications, an irregular or microlobulated margin, marked hypoechogenicity, and a shape that was more tall than it was wide as the criteria for malignant nodules and obtained $93.8 \%$ sensitivity and $66 \%$ specificity. A significant difference was not observed on length-to-width ratio using MDCT, which might attribute to technological disparity. Thus, it may be inferred that the MDCT features on solid composition and margin shape would be helpful for preoperative diagnosis for CTL patients.

The present findings differed from that of a previous study, which concluded that on diameter comparison, no significant association was found between malignancy and a nodule size of $>15 \mathrm{~mm}$ using FNAB (20). The higher incidence of microcarcinoma in the present study and timely surgery on suspected carcinomas may contribute to the difference in conclusions. Generally, the density is homogenous for small nodules, but the density is gradually heterogeneous and cystic changed as the size enlarged, which was also confirmed by the enhancement results in the present study. However, no significant differences were observed in the $\mathrm{CT}$ value of plain scan, enhancement degree at arterial or venous phase in CTL patients with benign or malignant nodules, indicating that the enhancement degree on the parenchyma demonstrated no significant value in differentiating benign from malignant nodules.

Traditionally, FANB has been recognized as the standard test to determine whether surgical removal of a detected nodule. Given the low risk for thyroid cancer, the technology of FNAB performed on all nodules detected by imaging was not feasible or advisable $(21,22)$. Thereafter, a serious of imaging modalities was developed, including ultrasound, carotid duplex scan, CT, magnetic resonance imaging (MRI) or positron emission tomography (PET). However, mixed results were reported using these imaging modalities. For example, Mitchell et al (23) reported that 18FDG-PET with sensitivity of $60 \%$ and a specificity of $91 \%$ based on 48 malignant lesions and 33 benign lesions, which performed similarly with sonographic diagnosis (91.4-92.5\% specificity) when the ratio of anteroposterior to transverse diameter ratio was $>1$. Basharat et al (24) has compared thyroid scan with FNAB and suggests fine needle aspiration cytology is more specific than sensitive whereas thyroid scan is more sensitive than specific in detecting thyroid malignancy. In 2011, a study reported the highest values for sensitivity and specificity based on (nodule/ cord SI)/nodule apparent diffusion coefficient ratio using DW-MRI limited on 44 patients with nodules (25). Despite the high values for sensitivity and specificity, a missed malignancy would cause huge damage for the patient. However, to establish general criteria for differentiating malignant nodules from benign nodules according to the different backgrounds of CTL patients is difficult. Therefore, CT features that can differentiate between benign and malignant nodules are required. 
Certain limitations of the present study should be discussed. Firstly, no gold standard for detecting nodules was designed in the study. Although we have defined the criteria for each feature through combining the previous studies $(12,15)$, the criteria have limited the rigorous of the result. Secondly, malignant and benign thyroid nodules were just confirmed by surgery, and no follow-up was performed. Therefore, the possibility that the benign nodule may develop into the malignant nodule could not be excluded. Moreover, the conclusion from the small sample size of the enrolled cases may limit the reliability of the conclusion, and MDCT characterization for benign and malign nodules on a larger sample size is needed.

In conclusion, the findings suggest that CLT patients with malignant nodules mainly present with features of solid composition, ill-defined margin, unclear or no capsule, or micro-calcifications, which could be used as the diagnosis method in advance for FANB. However, further study based on a larger sample size and cases with different backgrounds is required in order to confirm the above method is suitable to differentiate benign and malign nodules.

\section{References}

1. Anderson L, Middleton WD, Teefey SA, Reading CC, Langer JE, Desser T, Szabunio MM, Mandel SJ, Hildebolt CF and Cronan JJ: Hashimoto thyroiditis: Part 2, sonographic analysis of benign and malignant nodules in patients with diffuse Hashimoto thyroiditis. AJR Am J Roentgenol 195: 216-222, 2010

2. Anderson L, Middleton WD, Teefey SA, Reading CC, Langer JE, Desser T, Szabunio MM, Hildebolt CF, Mandel SJ and Cronan JJ Hashimoto thyroiditis: Part 1, sonographic analysis of the nodular form of Hashimoto thyroiditis. AJR Am J Roentgenol 195: 208-215, 2010.

3. Sakiyama R: Thyroiditis: A clinical review. Am Fam Physician 48: 615-621, 1993.

4. Mazokopakis EE, Tzortzinis AA, Dalieraki-Ott EI, Tsartsalis AN, Syros PK, Karefilakis CM, Papadomanolaki MG and Starakis IK: Coexistence of Hashimoto's thyroiditis with papillary thyroid carcinoma. A retrospective study. Hormones (Athens) 9: 312-317, 2010.

5. Zhang Y, Dai J, Wu T, Yang N and Yin Z: The study of the coexistence of Hashimoto's thyroiditis with papillary thyroid carcinoma. J Cancer Res Clin Oncol 140: 1021-1026, 2014.

6. Zosin I and Balaş M: Clinical, ultrasonographical and histopathological aspects in Hashimoto's thyroiditis associated with malignant and benign thyroid nodules. Endokrynol Pol 64: 255-262, 2013

7. Reading CC, Charboneau JW, Hay ID and Sebo TJ: Sonography of thyroid nodules: A ‘classic pattern' diagnostic approach. Ultrasound Q 21: 157-165, 2005.

8. Wang L, Xia Y, Jiang YX, Dai Q and Li XY: Likelihood ratio-based differentiation of nodular Hashimoto thyroiditis and papillary thyroid carcinoma in patients with sonographically evident diffuse hashimoto thyroiditis: Preliminary study. J Ultrasound Med 31: 1767-1775, 2012.
9. Gul K, Dirikoc A, Kiyak G, Ersoy PE, Ugras NS, Ersoy R and Cakir B: The association between thyroid carcinoma and Hashimoto's thyroiditis: The ultrasonographic and histopathologic characteristics of malignant nodules. Thyroid 20: 873-878, 2010.

10. Cronan JJ: Thyroid nodules: Is it time to turn off the US machines? Radiology 247: 602-604, 2008.

11. Frates MC, Benson CB, Charboneau JW, Cibas ES, Clark OH, Coleman BG, Cronan JJ, Doubilet PM, Evans DB, Goellner JR, et al: Management of thyroid nodules detected at US: Society of radiologists in ultrasound consensus conference statement. Radiology 237: 794-800, 2005.

12. Kim EK, Park CS, Chung WY, Oh KK, Kim DI, Lee JT and Yoo HS: New sonographic criteria for recommending fine-needle aspiration biopsy of nonpalpable solid nodules of the thyroid. AJR Am J Roentgenol 178: 687-691, 2002.

13. Yang GC, Liebeskind D and Messina AV: Ultrasound-guided fine-needle aspiration of the thyroid assessed by Ultrafast Papanicolaou stain: Data from 1135 biopsies with a two-to six-year follow-up. Thyroid 11: 581-589, 2001.

14. Ishigaki S, Shimamoto K, Satake H, Sawaki A, Itoh S, Ikeda M, Ishigaki T and Imai T: Multi-slice CT of thyroid nodules: Comparison with ultrasonography. Radiat Med 22: 346-353, 2004.

15. Kang HW, No JH, Chung JH, Min YK, Lee MS, Lee MK, Yang JH and Kim KW: Prevalence, clinical and ultrasonographic characteristics of thyroid incidentalomas. Thyroid 14: 29-33, 2004.

16. Anil C, Goksel S and Gursoy A: Hashimoto's thyroiditis is not associated with increased risk of thyroid cancer in patients with thyroid nodules: A single-center prospective study. Thyroid 20: 601-606, 2010.

17. Kim KW, Park YJ, Kim EH, Park SY, Park do J, Ahn SH, Park do J, Jang HC and Cho BY: Elevated risk of papillary thyroid cancer in Korean patients with Hashimoto's thyroiditis. Head Neck 33: 691-695, 2011.

18. Repplinger D, Bargren A, Zhang YW, Adler JT, Haymart M and Chen H: Is Hashimoto's thyroiditis a risk factor for papillary thyroid cancer? J Surg Res 150: 49-52, 2008.

19. Fiore E, Rago T, Latrofa F, Provenzale MA, Piaggi P, Delitala A, Scutari M, Basolo F, Di Coscio G, Grasso L, et al: Hashimoto's thyroiditis is associated with papillary thyroid carcinoma: Role of TSH and of treatment with L-thyroxine. Endocr Relat Cancer 18: 429-437, 2011.

20. Rahimi M, Farshchian N, Rezaee E, Shahebrahimi K and Madani H: To differentiate benign from malignant thyroid nodule comparison of sonography with FNAC findings. Pak J Med Sci 29: 77-80, 2013.

21. Lawrence W Jr and Kaplan BJ: Diagnosis and management of patients with thyroid nodules. J Surg Oncol 80: 157-170, 2002.

22. Khalid AN, Hollenbeak CS, Quraishi SA, Fan CY and Stack BC Jr: The cost-effectiveness of iodine 131 scintigraphy, ultrasonography, and fine-needle aspiration biopsy in the initial diagnosis of solitary thyroid nodules. Arch Otolaryngol Head Neck Surg 132: 244-250, 2006.

23. Mitchell JC, Grant F, Evenson AR, Parker JA, Hasselgren PO and Parangi S: Preoperative evaluation of thyroid nodules with 18FDG-PET/CT. Surgery 138: 1166-1175, 2005.

24. Basharat R, Bukhari MH, Saeed S and Hamid T: Comparison of fine needle aspiration cytology and thyroid scan in solitary thyroid nodule. Patholog Res Int 2011: 754041, 2011.

25. Mutlu H, Sivrioglu AK, Sonmez G, Velioglu M, Sildiroglu HO, Basekim CC and Kizilkaya E: Role of apparent diffusion coefficient values and diffusion-weighted magnetic resonance imaging in differentiation between benign and malignant thyroid nodules. Clin Imaging 36: 1-7, 2012. 